\section{New funding needed for late-stage vaccine work?}

Vaccine manufacturers, governments and charities need to start taking on the costs of late-stage development work if the next wave of candidate vaccines are ever going to make it to market, argue GlaxoSmithKline Vaccines' chief scientist Rino Rappuoli and his colleagues in academia in a recent perspective article in Science Translational Medicine.

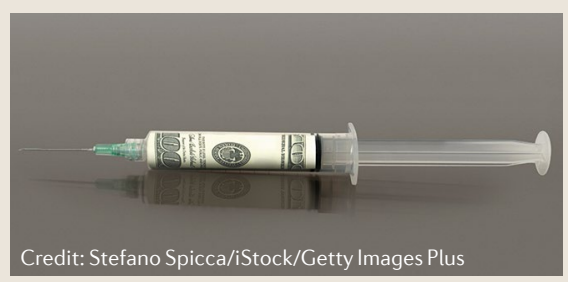

Vaccine development currently takes place over three stages, at a cost of over US $\$ 1$ billion, the authors write. The first two stages, discovery and early-stage development, account for around $30 \%$ of the overall costs and are backed collaboratively by industry, academia and philanthropic and government funders. Late-stage development, covering phase III trials and vaccine-production scale-up, accounts for the other $70 \%$, and is done nearly exclusively by large pharmaceutical firms.

Until now, industry has taken these costs on because it can sell vaccines at higher costs in developed countries to make up for low revenue from developing ones. But this model is breaking down, Rappuoli and colleagues write. Diseases including malaria, tuberculosis, Ebola and Zika virus are predominantly problematic in low- and middle-income countries, and dual-market strategies for vaccines against these diseases may offer little opportunity to recoup costs. Despite progress with candidate vaccines against these diseases, large pharmaceutical firms are increasingly losing interest in funding their late-stage development.

The authors propose several partnership models to address this growing gap, including direct funding for late-stage work from donors such as the Bill \& Melinda Gates Foundation or governmental bodies.

"We favor a partnership where large pharma is involved in early development, providing available technologies, intellectual property (IP), know-how, and early GMP manufacturing, as well as ushering the vaccine to clinical proof of concept alongside clinical partners. Once clinical proof of concept is achieved, large pharma can decide to carry on late-stage development on its own if a dual market is present. If not, then it could transfer the technology — relieving itself of late-stage development and marketing costs - to a new entity," they write.

The authors made similar arguments in a comment paper Nature late last year.

Asher Mullard

\section{Anti-CD3 antibody charts out a type 1 diabetes comeback}

Hope springs eternal for CD3-binding antibodies in type 1 diabetes, suggest new clinical data in the New England Journal of Medicine.

Researchers have been looking into the potential of immunosuppressive CD3-binding antibodies in type 1 diabetes for more than three decades. As early as 1989, clinicians had dosed patients with type 1 diabetes with an anti-CD3 antibody in the hope of slowing autoimmune $\beta$-cell death and preserving insulin production. This work eventually attracted the attention of several major pharmaceutical firms, but pivotal trials failed to deliver. Eli Lilly and MacroGenics reported in 2011 that their CD3-targeting teplizumab failed to meet its primary end point in a phase III trial in more than 500 patients with the disease. GlaxoSmithKline discontinued development of its otelixizumab, in the same setting, after it too failed in pivotal trials.

Now, an investigator-led trial of teplizumab for the prevention of disease onset offers another path forward. The phase II trial randomized 76 participants with high risk of developing disease - nondiabetic relatives of patients with type 1 diabetes, with signs of the earliest stages of disease - onto a 2-week course of teplizumab or placebo. The median time to the diagnosis of type 1 diabetes was 4 years in the teplizumab arm, compared with 2 years in the placebo arm, showed the $\mathrm{NIH}$-sponsored trial. "Our findings support the notion that type 1 diabetes is a chronic T-cell-mediated disease and suggest that immunomodulation before the development of clinical disease can be useful," the investigators wrote.

The study had several limitations including its small size and limited statistical power - but it provides a big boost for Provention Bio, which acquired rights to teplizumab in 2018. The company is evaluating a regulatory path forward for the antibody in at-risk individuals.

In April, Provention also started enrolling volunteers into a phase III trial of teplizumab in newly diagnosed patients aged $8-17$ years, a potentially more responsive patient population than Eli Lilly and MacroGenics explored in their pivotal trial of the antibody.

Asher Mullard

\section{Large pharmas set up screening library data-sharing experiment}

Although small-molecule screening libraries are sometimes called the 'crown jewels' of large pharmaceutical firms, companies are increasingly open to precompetitive projects that promise to make the most of these resources. AstraZeneca and Bayer opened their libraries to one another to increase their coverage of chemical space in 2011, for example, and then AstraZeneca and Sanofi exchanged 210,000 compounds in 2015. In the latest blockchain-enabled iteration of this strategy, ten large firms are now set to pool the data describing their chemical libraries so that the partners can build better computational models of drug activity.

Under MELLODDY — an €18-million, 3-year IMI project - partners will share data about the structure and activity of the compounds in their screening libraries, without ceding control or privacy over any of their intellectual property. Partners will be able to use this supersized data set to build and train predictive drug-screening models, but without being able to see the specifics of what their partners have contributed.

"By giving people the guarantee that their data is safe, and that no one else can directly look at it, it does lead to a very different mindset. We would have never previously thought of exercises where tens of millions of compounds, from ten pharmaceutical firms, could be involved in a single analysis," says Hugo Ceulemans, MELLODDY project leader and scientific director at Janssen.

The predictive models that companies can build with this data should be much better than what they can achieve using only their own internal data, he adds.

The large pharma partners in the project consist of Amgen, Astellas, AstraZeneca, Bayer, Boehringer Ingelheim, GlaxoSmithKline, Janssen, Merck KgaA, Novartis and Servier.

Asher Mullard 